\title{
Distant BCG disease in a child with suspicion of Hyperimmunoglobulin E Syndrome
}

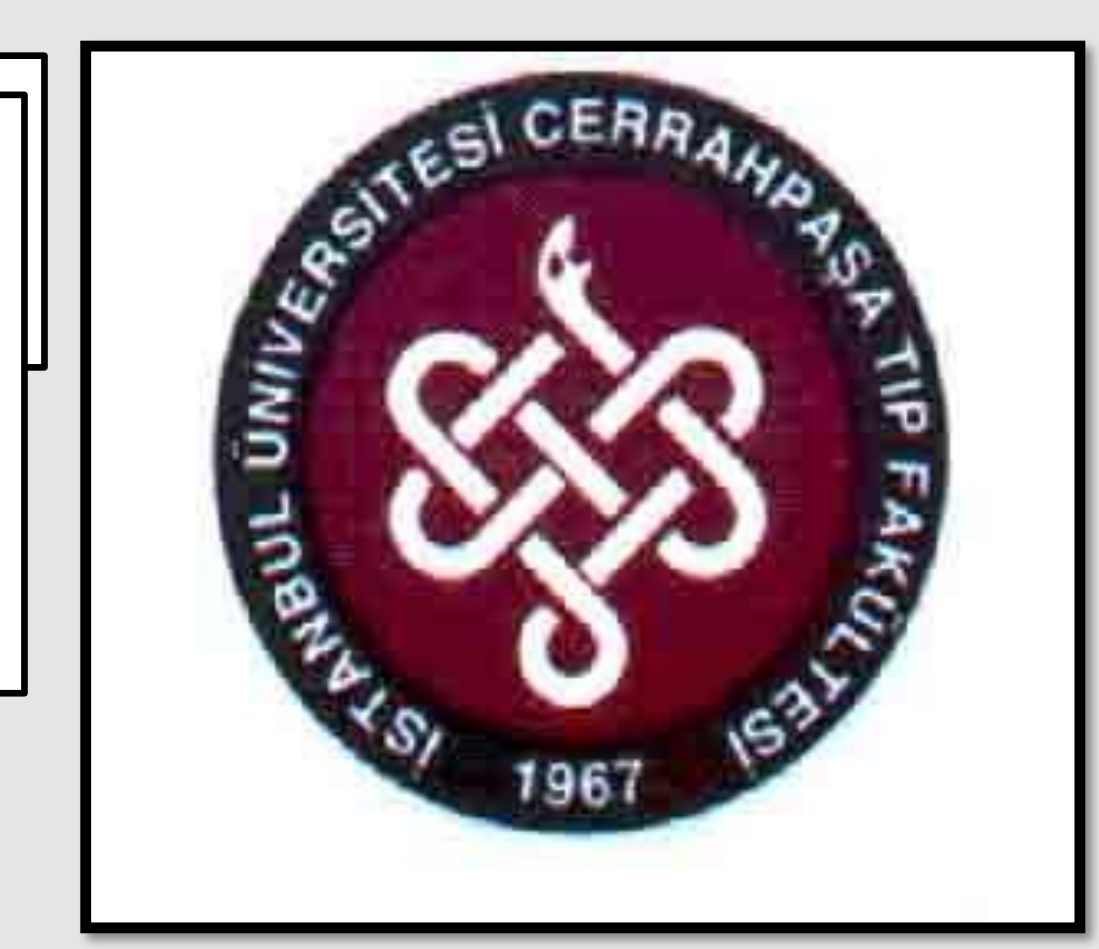

Aygun D.(1), Belhan E.(2), Nepesov S (1), Camcioglu, Y.(1)

1 Istanbul University ,Cerrahpasa Medical Faculty ,Department of Pediatric Infectious Disease 2Istanbul University ,Cerrahpasa Medical Faculty ,Department of Pediatric Intensive Care

\section{Introduction}

The bacilli Calmette-Guérin (BCG) vaccine is included in the national childhood immunization program and administered to all newborns in countries where tuberculosis is endemic. The BCG vaccination is safe and severe adverse effects are extremely rare in immunocompetent children. However, immunocompromised hosts like primary immunodeficiencies (PID) are prone to complications of vaccine. PID is usually not diagnosed at the time of BCG vaccination, so can been associated with severe complications. Primary immune-deficiencies like severe combined immune-deficiency and chronic granulo-matous disease were diagnosed in $25-50 \%$ of children. Complications of BCG vaccine are collectively known as BCG disease. BCG confirmed from one site beyond a local or regional ipsilateral process like pulmonary secretions (gastric or tracheal aspirate) is called distant BCG disease. Herein, we report a child with PID who had developed distant BCG disease. Mycobacterium bovis was isolated both in gastric aspirate and vaccine site.

\section{Case}

A three year old girl admitted with lymphadenitis having purulent discharge. She had the history of failure to thrive, eczema, recurrent perianal abscess, recurrent pneumonia and pneumotocele. She was born from non-consanguineous couple, she was only vaccinated with BCG vaccine. On physical examination, she had colostomy bag, perianal fistula and a large lymphadenitis in $5 \times 5 \mathrm{~cm}$ diameter on left arm at the localisation of BCG scar. Mycobacterium bovis was isolated in the lymphadenitis exudate and gastric aspirate. The microorganism had isoniazid and pyrazinamide resistance (pyrazinamide having native resistance). The chest radiography showed the previous pneumotocele with no sign of tuberculosis and osteomyelitis was excluded. The immunological evaluation revealed Ig $315 \mathrm{mg} / \mathrm{dl}$, Ig A $15 \mathrm{mg} / \mathrm{dl}, \operatorname{IgM} 40 \mathrm{mg} / \mathrm{dl}$, IgE >5000 $\mathrm{IU} / \mathrm{ml}$ and lymphocyte subset analysis was normal. Hyperimmunoglobulin E Syndrome (HIES) scoring system was 40 and was considered as indeterminate HIES.

\section{Conclusion}

BCG dissemination in a child with the recurrent infections may be the presenting clinical picture of a PID and may be associated with a disseminated disease. Severe combined immunodeficiency, DiGeorge syndrome, chronic granulomatous disease, and defective CD40-CD40L signaling (CD40L, CD40, NEMO, and IкB- $\alpha$ mutations) are the immunodeficiencies that have broader vulnerability to the BCG vaccine. The diagnosis of our patient is not clear yet but HIES can yield to BCG disease since it is a PID. 